\title{
VI. Zusammenfassende Schlussbetrachtungen
}

Im Zentrum der öffentlichen Diskussion um die EMRK stand von allem Anfang an die Suche nach einem Ausgleich zwischen den vermeintlichen Antagonismen eines effektiven internationalen Menschenrechtsschutzes und nationaler Souveränität. ${ }^{1}$ Bei der Ausarbeitung der EMRK wurde in diesem Sinne insbesondere diskutiert, wie sich die Schaffung eines internationalen Rechtsschutzmechanismus auf den bis dahin vorrangig nationalen Menschenrechtsschutz auswirken würde. In der Schweiz beispielsweise befürchteten Gegnerinnen eines EMRK-Beitritts die Überlagerung der bundesgerichtlichen Rechtsprechung zu den Grundrechten und damit eine „Entthronung von Lausanne“. ${ }^{2}$ Befürchtet wurde in der Schweiz und andernorts aber auch eine unzweckmäßige Einengung der Entscheidungsbefugnisse nationaler Verfassungs- und Gesetzgebungsorgane. ${ }^{3}$ Kurz: Schon in der Phase der Ausarbeitung beschäftigte die Frage, welche normativen Implikationen das institutionelle Arrangement der EMRK für die europäischen Grundrechtsordnungen zeitigen würde. Diese Diskussion ist seither nie mehr abgerissen, sondern hat wohl noch an Bedeutung gewonnen. Seit einigen Jahren wird unter zunehmender Referenzierung des Subsidiaritätsgrundsatzes insbesondere debattiert, wie der EGMR die EMRK angesichts seiner besonderen Stellung auslegen soll. ${ }^{4}$ Nur am Rande thematisiert wird hingegen die an sich logisch vorgelagerte Frage, welchen Sachverhalt er seinen Urteilen zugrunde legen soll.

\footnotetext{
${ }^{1}$ Vgl. dazu und zum Folgenden Christoffersen, S. 7 ff.

${ }^{2}$ Vgl. Kreis, S. 62 f. Vgl. zur Diskussion in der Schweiz vor Beitritt zur EMRK außerdem Seiler, S. 224.

${ }^{3}$ Vgl. zur Diskussion im Vereinigten Königreich Thurnheer, S. 135 ff.

${ }^{4}$ Vgl. statt vieler Arai-Takahashi; Legg.
} 
Diese Schwerpunktsetzung hängt damit zusammen, dass die Rechtsprechung des EGMR nur schwer überblickbar ist. Entsprechend wird in der Doktrin zur EMRK auf Straßburger Urteile fokussiert, die gesellschaftspolitisch von großer Tragweite sind. ${ }^{5}$ In den Mittelpunkt des Interesses gerückt werden dabei insbesondere die normativen Implikationen dieser Urteile für die europäischen Grundrechtsordnungen; konkret entschiedene Sachverhalte und damit auch die Frage der Tatsachenfeststellung rücken demgegenüber in den Hintergrund. Wenig Beachtung gefunden hat die Frage der Tatsachenfeststellung davon abgesehen aber auch deshalb, weil der Sachverhalt in den Verfahren vor dem EGMR vor noch nicht allzu langer Zeit nur selten umstritten war. ${ }^{6}$ Eine Analyse neuerer Urteile legt jedoch nahe, dass sich an diesem Bild einiges geändert hat: Gerade unter Art. 3 EMRK streiten sich die Parteien heute oftmals weniger über die Auslegung der Konventionsgarantien als über die Frage, was sich in Tat und Wahrheit zugetragen hat. Die Frage der Tatsachenfeststellung rückt so in den Mittelpunkt des Interesses von Lehre und Rechtsprechung.

Die Verschiebung des Interesses ist zu begrüßen, denn die Bedeutung der Tatsachenfeststellung für einen effektiven Schutz der Konventionsgarantien kann gar nicht unterschätzt werden. ${ }^{7}$ Wie alle individualrechtlich ausgestalteten Rechtsschutzmechanismen basieren die nationalen und internationalen Gerichtsverfahren zum Schutz der Konventionsrechte auf einer beweisrechtlich gesteuerten Feststellung der maßgeblichen Tatsachen. Primärer Zweck eines jeden Beweisrechts ist es dabei, die effektiven Gegebenheiten und Geschehnisse möglichst objektiv zu rekonstruieren und damit das Risiko einer fehlerhaften Rechtsanwendung auf ein Minimum zu reduzieren. Weil Gerichte durch ihre Urteile eine zumindest juristische Wahrheit konstruieren, sollen sie sämtliche für ihre Entscheidung erheblichen Tatsachen kennen. Diese Wahrheitsermittlungsfunktion des Beweisrechts hat eine besondere Bedeutung, wenn ein Gericht Vorgänge beurteilt, bei denen die Einzelperson der Staatsgewalt mehr oder weniger schutzlos ausgeliefert war: Angesichts des Machtgefälles zwischen dem Individuum und den mit Zwangsbefugnissen ausgestatteten staatlichen Organen besteht in diesem Zusammenhang nämlich die besondere Gefahr, dass auf Kosten individueller Rechte wahrheitsverzerrende staatliche Narrative konstruiert werden. ${ }^{8}$ Es ist eine der zentralen Funktionen der EMRK, für solche Konstellationen einen griffigen überstaatlichen Rechtsschutz zu schaffen. ${ }^{9}$

\footnotetext{
${ }^{5}$ So beispielsweise das Urteil zum französischen Burkaverbot, das auch in der Schweiz starke Beachtung gefunden hat; vgl. EGMR (Große Kammer), Urteil vom 1. Juli 2014, S.A.S. v. Frankreich. ${ }^{6}$ Vgl. Drzemczewski, S. 124.

${ }^{7} \mathrm{Vgl}$. dazu und zum Folgenden oben, II. A.

${ }^{8}$ Vgl. zum Missbrauch des Terrorismusnarrativs zur Diskreditierung von (politischen) Gegnerinnen und Gegnern Jackson, S. 394 ff.

${ }^{9}$ Vgl. Wildhaber, Europäischer Grundrechtsschutz, S. 689, der von einem „letzten Auffangnetz“ spricht.
} 
Der von der EMRK bezweckte effektive Menschenrechtsschutz verlangt also, dass behauptete Konventionsverletzungen angemessen untersucht werden. ${ }^{10}$ In diesem Sinne hat der EGMR in seiner jüngeren Rechtsprechung aus den materiellen Konventionsgarantien und Art. 13 EMRK (zuletzt auch unter dem Stichwort eines „Rechts auf Wahrheit" ${ }^{11}$ zahlreiche prozedurale Vorgaben entwickelt, welche den nationalen Behörden und Gerichten Untersuchungspflichten überbürden. Anderseits nehmen Tatfragen auch in den Urteilen des EGMR immer mehr Raum ein. Die Rechtsprechung des EGMR widerspiegelt insofern die wachsende Bedeutung der Tatsachenfeststellung: Gerade wo es um die menschenrechtlich geschützten Kernbereiche der Persönlichkeit geht, darf auch in Zeiten von fake news kein Platz bestehen für einen Realitätsbegriff, der lediglich an ,überzeugende Kommunikation und glaubwürdig erzählte Geschichten" anknüpft. ${ }^{12}$

Zwischen den konventionsrechtlich statuierten Untersuchungspflichten der innerstaatlichen Behörden und der Tatsachenfeststellung auf Ebene des EGMR besteht dabei ein engerer rechtlicher Zusammenhang, als dies auf den ersten Blick erscheinen mag. Zwar ist der EGMR völkerrechtlich konstituiert und kann im Verhältnis zu den nationalen Gerichten schon deshalb nicht als Instanzengericht betrachtet werden. Insofern kann auch seine Sachverhaltskognition nicht durch nationales Verfahrensrecht eingeschränkt sein. Weil dem Verfahren vor dem EGMR aber aufgrund des Ausschöpfungsgrundsatzes (Art. 35 Ziff. 1 EMRK) fast immer ein nationales Verfahren vorangeht, sind die nationalen Instanzen auch im Bereich der Tatsachenfeststellung mit dem EGMR verbunden. Meist bewegt sich die Sachverhaltsfeststellung des EGMR also nicht im „luftleeren Raum“; vielmehr kann der EGMR auf die Feststellungen nationaler Gerichte zurückgreifen. Die seit jeher diskutierte Frage des Verhältnisses zwischen nationalen Behörden und Gerichten einerseits und dem EGMR anderseits stellt sich also nicht nur mit Blick auf die materielle Auslegung der Konventionsgarantien, sondern auch bezüglich der im Einzelfall vorgelagerten Feststellung der maßgeblichen Tatsachen. Es ist diese zuletzt angesprochene Frage des Verhältnisses zwischen nationalen Gerichten und EGMR bezüglich der Tatsachenfeststellung, die vorliegend Gegenstand der Untersuchung war.

Zur Klärung dieser Frage wurde in der vorliegenden Arbeit aufgrund des Fehlens konkreterer Bestimmungen auf den Subsidiaritätsgrundsatz zurückgegriffen. ${ }^{13}$ Diese dogmatische Fundierung rechtfertigt sich, weil allgemein anerkannt ist, dass der Subsidiaritätsgrundsatz im Sinne eines „Grundbausteins“ die gesamte

\footnotetext{
${ }^{10} \mathrm{Vgl}$. dazu und zum Folgenden oben, II. B.

${ }^{11}$ Vgl. EGMR (Große Kammer), Urteil vom 13. Dezember 2010, El-Masri v. Mazedonien, § 191.

${ }^{12}$ Ein solcher Wahrheitsbegriff scheint beispielsweise in der folgenden, vom Evolutionsbiologen Barnaby Marsh formulierten „Edge Question“ auf: „How much of what we call ,reality“ is ultimately grounded and instantiated in convincing communication and storytelling?“. Vgl. zu dieser und anderen „Edge Questions“ https://www.edge.org/the-last-question-8. Zugegriffen am 01.03.2018.

${ }^{13} \mathrm{Vgl}$. oben, II. C. 2.
} 
Rechtsprechungstätigkeit des EGMR durchzieht und insbesondere über dessen Verhältnis zu den nationalen Institutionen bestimmt. Gleichzeitig ist die Anknüpfung an den Subsidiaritätsbegriff schwierig, weil in der Diskussion um die EMRK und auch in der Rechtsprechung des EGMR der dogmatische Gehalt des Grundsatzes nicht immer klar fassbar ist. Zur Präzisierung des Gehalts des Subsidiaritätsgrundsatzes unter der EMRK wurde vorliegend ein vergleichender Ansatz gewählt: In der Kontrastierung zu anderen dogmatischen Ausprägungen des Subsidiaritätsgrundsatzes sollten gleichsam die Konturen des konventionsrechtlichen Subsidiaritätsprinzips deutlicher herausgestrichen werden. ${ }^{14}$ Im Rechtsvergleich sind dabei namentlich zwei Dimensionen des Subsidiaritätsgrundsatzes zu unterscheiden: Ursprünglich diente er vorrangig dem Schutz des Individuums und grenzte den persönlichen Einflussbereich von demjenigen der Gesellschafts- und Staatstätigkeit ab. Gebräuchlich ist heute überdies vor allem die Verwendung des Begriffs im interinstitutionellen - und namentlich bundesstaatlichen - Kontext. In diesem letzteren Zusammenhang bezweckt der Subsidiaritätsgrundsatz in erster Linie die zweckmäßige Abgrenzung der Kompetenzbereiche verschiedener miteinander verbundener Gemeinwesen.

Intuitiv liegt auf der Hand, dass dem Subsidiaritätsgrundsatz in der EMRK eine interinstitutionelle Bedeutung zukommt: ${ }^{15}$ Auch in der EMRK stehen sich mit dem EGMR einerseits und den Behörden der Mitgliedstaaten anderseits nämlich eine „umfassende“ und eine „umfasste Ebene“ gegenüber. Die beiden Ebenen sind auf das gemeinsame Ziel eines effektiven Schutzes der materiellen EMRK-Garantien ausgerichtet und besitzen insoweit einen gemeinsamen Aufgabenkreis. Das Subsidiaritätsprinzip unter der EMRK bezweckt ein Zusammenspiel zwischen mitgliedstaatlichen Institutionen und EGMR, das dem solchermaßen vorgegebenen Verbundzweck gerecht wird, ohne in dysfunktionaler Art und Weise die Handlungsbefugnisse der Konventionsstaaten als primär zuständiger Ebene einzuschränken. Der wirksame europäische Grundrechtsschutz ist also gewissermaßen der Leitstern, den die Interpretin der EMRK immer im Auge behalten muss, um dem Subsidiaritätsgrundsatz einen fassbaren Gehalt zu verleihen. Anders als im bundesstaatlichen Kontext adressiert der konventionsrechtliche Subsidiaritätsgrundsatz mit dem EGMR auf der „umfassenden Ebene“ jedoch ein Gericht und kein Legislativorgan. Als Gericht entscheidet der EGMR Einzelfälle; im Unterschied zu den sonst angesprochenen Rechtsetzungsorganen fehlt ihm die Befugnis, abstrakt eine Kompetenzordnung zu erlassen. Eine Definition des Subsidiaritätsgrundsatzes in Anlehnung an die im bundesstaatlichen Kontext dominierende Kompetenzabgrenzungsfunktion ist für die EMRK deshalb unbrauchbar. Rechtliche Bedeutung hat der Subsidiaritätsgrundsatz unter der EMRK vielmehr als Kompetenzausübungsregel, die sich in erster Linie an den EGMR richtet, mittelbar aber auch auf das innerstaatliche Verfahren durchschlägt. Inhaltlich verlangt der Subsidiaritätsgrundsatz, dass der EGMR in seiner Rechtsprechungstätigkeit seine

\footnotetext{
${ }^{14} \mathrm{Vgl}$. dazu und zum Folgenden oben, III. A.

${ }^{15} \mathrm{Vgl}$. dazu und zum Folgenden oben, III. B.
} 
besondere Stellung als internationales Gericht berücksichtigt und nur einschreitet, wenn und soweit die nationalen Behörden ihrer Primärverpflichtung zum Schutz der Konventionsrechte nicht nachgekommen sind oder nicht nachkommen konnten. Zurückhaltung ist dabei insbesondere angezeigt, wenn die nationalen Instanzen besser geeignet sind, eine bestimmte konventionsrechtlich vorgesehene Funktion auszuüben.

Gerade für die konventionsrechtliche Teilaufgabe der Tatsachenfeststellung sind die nationalen Instanzen dabei aufgrund ihrer Nähe zum Einzelfall deutlich besser ausgestattet als der EGMR. ${ }^{16}$ Die größere Nähe zum Einzelfall manifestiert sich dabei in vielfältiger Art und Weise: Beispielsweise finden im innerstaatlichen Verfahren oftmals Befragungen der Parteien und Anhörungen allfälliger Zeuginnen statt; auf der Ebene des EGMR kommt es jedoch nur in Einzelfällen zu Einvernahmen und dem EGMR fehlt insoweit der persönliche Eindruck der Streitbeteiligten. Auch sonst ist der EGMR nur beschränkt in der Lage, zur Klärung des maßgeblichen Sachverhalts ein Beweisverfahren durchzuführen. Neben den limitierten personellen Ressourcen spielt dabei insbesondere eine Rolle, dass das Verfahren vor dem EGMR in der Regel erst Jahre nach dem beanstandeten Vorfall angestoßen wird und bestimmte Beweise zu diesem Zeitpunkt gar nicht mehr erhoben werden können. Der EGMR entscheidet daher vielfach auf Grundlage jener Akten, die schon im innerstaatlichen Verfahren vorlagen. Soweit diese Akten weder in Englisch noch in Französisch abgefasst sind, tritt für den EGMR zusätzlich zu seinen beschränkten Beweiserhebungsmöglichkeiten ein Sprachenproblem auf: Angesichts der Vielfalt der Amtssprachen der Europaratsstaaten verstünde oftmals nur eine Minderheit des urteilenden Spruchkörpers die maßgeblichen innerstaatlichen Akten, so dass auch die von den nationalen Gerichten abweichende Würdigung von Beweismitteln besonderer Begründung bedarf.

Weil die innerstaatlichen Instanzen also im Grundsatz besser geeignet sind, die konventionsrechtlich maßgeblichen Tatsachen festzustellen, spricht der Subsidiaritätsgrundsatz für einen diesbezüglichen Handlungsvorrang der innerstaatlichen Behörden und Gerichte. ${ }^{17}$ Umgekehrt müssen die nationalen Institutionen ihrer diesbezüglichen Aufgabe im Sinne einer Primärverpflichtung aber auch tatsächlich nachkommen, um dem Verbundzweck Genüge zu tun. Versäumen sie in der Beurteilung des Einzelfalls eine methodisch nachvollziehbare Sachverhaltsfeststellung, entsteht subsidiaritätsrechtlich gesprochen jenes Vakuum, das der EGMR ausfüllen muss, um einen effektiven Menschenrechtsschutz zu gewährleisten. Grundsätzlich greift der EGMR im Einklang mit diesem subsidiaritätsrechtlichen Grundgedanken nur in Ausnahmefällen in die innerstaatlichen Tatsachenfeststellungen ein. Solche Ausnahmefälle liegen gemäß der Rechtsprechung einerseits vor, wenn ,zwingende Gründe“ eine Korrektur der innerstaatlichen Tatsachenfeststellungen gebieten, anderseits, wenn die Umstände des Einzelfalls als so ,,außergewöhnlich“ zu werten sind, dass sie

\footnotetext{
${ }^{16} \mathrm{Vgl}$. dazu und zum Folgenden oben, III. B. 4. a.

${ }^{17} \mathrm{Vgl}$. dazu und zum Folgenden oben, III. B. 4. b.
} 
ein Tätigwerden des EGMR als „erstinstanzliches Tatsachengericht“ erforderlich machen.

Heruntergebrochen auf den konkreten Einzelfall bedeutet dies, dass der EGMR seine „Tatsachenkognition“ maßgeblich von der Qualität der innerstaatlichen Sachverhaltsfeststellungen abhängig machen muss. ${ }^{18}$ Maßstab für die Qualität des innerstaatlichen Verfahrens bilden dabei die zahlreichen prozeduralen Garantien, die der EGMR in seiner neueren Rechtsprechung aus Art. 13 EMRK und aus verschiedenen materiellen Konventionsgarantien abgeleitet hat. ${ }^{19}$ Nach diesen konventionsrechtlichen Vorgaben sind innerstaatlich alle angemessenen verfahrensrechtlichen Schritte in die Wege zu leiten, die zur Erhellung des rechtserheblichen Sachverhalts beitragen können; zudem sind die innerstaatlichen Urteile (auch) in Bezug auf den maßgeblichen Sachverhalt angemessen zu begründen. ${ }^{20}$ Es besteht kein Grund, diese Vorgaben nur auf Fälle zu Art. 3 EMRK zu beschränken, zumal sich das vom EGMR entwickelte „Recht auf Wahrheit“" ausdrücklich auf Art. 13 EMRK und damit auf ein allgemeines, auf alle materiellen Konventionsgarantien anwendbares Verfahrensgrundrecht stützt.

Wenn die innerstaatlichen Behörden ihr Verfahren in Einklang mit den konventionsrechtlichen Vorgaben durchgeführt haben, darf der EGMR nur in Ausnahmefällen in deren Tatsachenfeststellungen eingreifen. ${ }^{21}$ Wie die Auswertung der EGMR-Praxis zu Art. 3 EMRK zeigt, verwendet der EGMR in dieser Konstellation zumindest implizit oft tatsächlich einen Willkürmaßstab. Die dogmatische Fundierung dieses Maßstabs im konventionsrechtlichen Subsidiaritätsgrundsatz legt nahe, dass eine Abweichung des EGMR vom innerstaatlich festgestellten Sachverhalt ganz allgemein - und nicht nur in Fällen zu Art. 3 EMRK - ,strong reasons" ${ }^{22}$ voraussetzt, wenn die prozeduralen Gehalte der EMRK im nationalen Verfahren beachtet worden sind. Umgekehrt rechtfertigt der Subsidiaritätsgrundsatz aber auch eine umfassende de-novo-Prüfung der innerstaatlich festgestellten Tatsachen, wenn die nationalen Behörden den prozeduralen Garantien der EMRK im innerstaatlichen Verfahren nicht gefolgt sind. ${ }^{23}$ In diesem Fall ist der EGMR nicht nur berechtigt, sondern sogar verpflichtet, sämtliche ihm vorliegenden Beweismittel im Hinblick auf die in Frage stehende Konventionsverletzung einer neuerlichen Prüfung zuzuführen. Die Anwendung spezifischer Beweisinstrumente - beispielsweise der Beweislastumkehr - erscheint angesichts der eingeschränkten Beweiserhebungsmöglichkeiten des EGMR als einzig denkbarer Weg, um den effektiven Schutz der Konventionsrechte trotz der Unzulänglichkeiten des

\footnotetext{
${ }^{18} \mathrm{Vgl}$. dazu oben, IV. A.

${ }^{19} \mathrm{Vgl}$. dazu und zum Folgenden oben, IV. B.

${ }^{20} \mathrm{Vgl}$. dazu oben, IV. B. 2. c.

${ }^{21}$ Vgl. dazu und zum Folgenden oben, IV. C. 1.

${ }^{22}$ Entwurf zu einer Kopenhagen-Deklaration, Ziff. 24.

${ }^{23} \mathrm{Vgl}$. dazu und zum Folgenden oben, IV. C. 2.
} 
innerstaatlichen Verfahrens sicherzustellen und das Risiko einer unzutreffenden Tatsachenfeststellung unter den Parteien angemessen zu verteilen. ${ }^{24}$

Gesondert zu betrachten ist die Frage, ob neue Tatsachenvorbringen durch den EGMR berücksichtigt werden dürfen. Im Hinblick auf den Normzweck des Ausschöpfungsgrundsatzes (Art. 35 Ziff. 1 EMRK) ist diesbezüglich ein maßgeblicher Unterschied zu machen zwischen Tatsachen und Beweismitteln, die schon zum Zeitpunkt des letzten innerstaatlichen Entscheids Bestand hatten (unechte Noven) und solchen, die erst danach entstanden sind (echte Noven). ${ }^{25}$ Während es einer Beschwerdeführerin im ersten Fall nämlich grundsätzlich möglich war, die zuständigen innerstaatlichen Gerichte mit dem konventionsrechtlich maßgeblichen Sachverhalt zu konfrontieren, ist diese Möglichkeit für den zweiten Fall zu verneinen. Der EGMR tritt auf Beschwerden, die gestützt auf unechte Noven einen völlig neuen Sachverhalt geltend machen, wohl auch aus dieser Überlegung oftmals gar nicht erst ein; dies gilt zumindest dann, wenn ein effektives Rechtsmittel zur Verfügung stand, mit dem der neu vorgebrachte Sachverhalt den innerstaatlichen Instanzen hätte unterbreitet werden können. ${ }^{26}$

Anders handhabt der EGMR mitunter echte Noven: Unter Berufung auf den Leitgedanken der EMRK, einen effektiven Menschenrechtsschutz zu gewährleisten, berücksichtigt er diese, wenn und soweit sie (behauptete) Konventionsverletzungen betreffen, die während des Verfahrens vor dem EGMR weiterhin latent wirksam waren, beziehungsweise in naher Zukunft wirksam zu werden drohen. ${ }^{27}$ Häufigstes Beispiel für eine solche Situation sind die zahlreichen Abschiebungsfälle, in denen die nationale Abschiebungsverfügung zwar rechtskräftig geworden ist, der Vollzug jedoch noch aussteht: Hier prüft der EGMR ex nunc, ob mit einer Abschiebung das ernsthafte Risiko einer unmenschlichen Behandlung einherginge. Er berïcksichtigt also auch Tatsachen, von denen die nationalen Gerichte zum Zeitpunkt ihres Entscheids gar nicht wissen konnten. Diese Praxis ist nach hier vertretener Auffassung subsidiaritätsrechtlich zu undifferenziert: ${ }^{28}$ Wo nämlich innerstaatliche Verfahren bestehen, die es erlauben würden, erhebliche nachträgliche Veränderungen der Sachlage einer innerstaatlichen Prüfung zuzuführen, besteht kein Vakuum, das der EGMR ausfüllen müsste. Die Existenz innerstaatlicher Wiedererwägungsverfahren ist dabei nicht nur eine Angelegenheit des nationalen Verfahrensrechts. Die in der Schweiz und anderswo bestehenden Wiedererwägungsverfahren bilden vielmehr die Vorgaben ab, die sich für die Konventionsstaaten aus der Konvention selbst ergeben: Namentlich Art. 1 EMRK und Art. 13 EMRK verlangen von den Konventionsstaaten nämlich, Verfahrenswege bereitzustellen, die eine Prüfung und allfällige Beseitigung behaupteter Konventionsverletzungen

\footnotetext{
${ }^{24}$ Vgl. dazu oben, IV. C. 3.

${ }^{25} \mathrm{Vgl}$. dazu oben, V. A.

${ }^{26} \mathrm{Vgl}$. dazu oben, V. C.

${ }^{27} \mathrm{Vgl}$. dazu und zum Folgenden oben, V. B.

${ }^{28} \mathrm{Vgl}$. dazu und zum Folgenden oben, V. D.
} 
schon auf innerstaatlicher Ebene erlauben. Der EGMR könnte deshalb zunächst untersuchen, ob innerstaatliche Verfahrenswege bestehen, mit welchen eine Beschwerdeführerin echte Noven einer effektiven Prüfung durch die nationalen Behörden zuführen könnte. Soweit dies zu bejahen wäre, müsste der EGMR die echten Noven in seinem Verfahren im Grundsatz ausblenden.

Open Access Dieses Kapitel wird unter der Creative Commons Namensnennung 4.0 International Lizenz (http://creativecommons.org/licenses/by/4.0/deed.de) veröffentlicht, welche die Nutzung, Vervielfältigung, Bearbeitung, Verbreitung und Wiedergabe in jeglichem Medium und Format erlaubt, sofern Sie den/die ursprünglichen Autor(en) und die Quelle ordnungsgemäß nennen, einen Link zur Creative Commons Lizenz beifügen und angeben, ob Änderungen vorgenommen wurden.

Die in diesem Kapitel enthaltenen Bilder und sonstiges Drittmaterial unterliegen ebenfalls der genannten Creative Commons Lizenz, sofern sich aus der Abbildungslegende nichts anderes ergibt. Sofern das betreffende Material nicht unter der genannten Creative Commons Lizenz steht und die betreffende Handlung nicht nach gesetzlichen Vorschriften erlaubt ist, ist für die oben aufgeführten Weiterverwendungen des Materials die Einwilligung des jeweiligen Rechteinhabers einzuholen. 